04

\title{
Усиление непотенциальных волн в замагниченной пучково-плазменной системе с учетом нестационарности плазмы
}

\author{
() И.Н. Карташов, М.В. Кузелев \\ Московский государственный университет, физический фракультет, \\ 119991 Москва, Россия \\ e-mail: igorkartashov@mail.ru, kuzelev@mail.ru
}

(Поступило в Редакцию 27 декабря 2017 г.)

Исследована пучковая неустойчивость с учетом непотенциальных эффектов в полностью замагниченной плазме, плотность которой медленно меняется с течением времени. Показано, что в усиливаемом сигнале (при распространении в такой электродинамической системе) появляется сдвиг частоты и получены аналитические соотношения, устанавливающие величину этого сдвига.

DOI: $10.21883 /$ JTF.2018.08.46306.2622

К настоящему времени в экспериментах по плазменной СВЧ электронике реализованы импульсные плазменные источники СВЧ излучения большой мощности со значительным диапазоном перестройки рабочей частоты (в 2 раза): плазменные усилители СВЧ $[1,2]$ и плазменные генераторы СВЧ $[3,4]$. Несмотря на это, имеется и целый ряд „вечных“ проблем, таких, как повышение мощности и увеличение длительности импульса излучения. Поэтому исследования, касающиеся различных аспектов пучково-плазменного взаимодействия, в настоящее время проводятся как экспериментально [1-5], так и теоретически [6-9]. Принцип действия плазменных источников СВЧ излучения основан на возбуждении электронным пучком, пронизывающим волновод, замедленных волн электродинамической системы. В плазменной СВЧ электронике в качестве замедляющей электродинамической системы используется волновод, заполненный плазмой. Вся система обычно помещается в достаточно сильное магнитное поле, замагничивающее электроны пучка и плазмы, и, таким образом, фокусирующее электронный пучок, который формируется в результате ускорения эмитированных со взрывоэмиссионного катода электронов. Плазма создается в результате развития пучково-плазменного разряда, при этом для ее создания используется вспомогательный электронный пучок. Время существования плазмы в реальных СВЧ излучателях превышает $100 \mu \mathrm{s}$ и при характерных длительностях импульса несколько сотен наносекунд плазма не успевает сколь-нибудь заметным образом рекомбинировать. Однако, как показывают исследования, плотность плазмы все же может меняться в течение импульса за счет ее ухода из рабочей области в процессе инжекции сильноточного электронного пучка [10]. Кроме того, при неполной ионизации плазмы возможна и ее дополнительная ионизация в процессе развития неустойчивости [2-4]. Настоящая работа посвящена исследованию пучково-плазменных неустойчивостей в нестационарных плазменных системах. В реальных системах нестационарной оказывается, в первую очередь, плотность плазмы, причем характерное время изменения плотности плазмы сушественно превышает период возбуждаемых колебаний.

Возбуждение и распространение волн в нестационарной среде исследовались и ранее. Хорошо известен такой механизм излучения электромагнитных волн равномерно движущимся зарядом в неоднородной среде, как переходное излучение, впервые исследованное в [11]. Аналогичное явление имеет место и в однородной среде, но с зависящим от времени показателем преломления [12-14]. Исследовались нестационарные процессы и при рассмотрении вопросов распространения и отражения электромагнитных волн оптического диапазона. Диэлектрическая проницаемость среды в ряде случаев меняется со временем. Это изменение может быть связано с процессами релаксации в среде, с лазерной накачкой среды или ее ионизацией внешним источником. Причем характерные времена изменения диэлектрической проницаемости могут быть как велики по сравнению с периодом волны (медленное изменение), так и сопоставимы с ним или меняться скачком (достаточно быстро по сравнению с периодом поля). Для рассмотрения с единых позиций всей совокупности характерных времен изменения свойств среды в работах $[15,16]$ исследование ведется на основе точного решения уравнений Максвелла для специально подобранных зависимостей диэлектрической проницаемости от времени. В частности, в работе [17] определяется изменение частоты сигнала, отраженного от неподвижной нестационарной среды. Кроме того, как нестационарные могут рассматриваться и иные случаи распространения, отражения или возбуждения волн, такие, как отражение от движущегося фронта ионизации или развитие пучково-плазменной неустойчивости в системах с коротким импульсом электронного пучка [18].

Рассмотрим холодную однородную электронную плазму. Невозмущенную плотность электронов $n_{0}(t)$ считаем медленно меняющейся функцией времени. Плазму пронизывает однородный электронный пучок, движущийся 
с постоянной скоростью $u$, направление которой выберем вдоль оси $z$. Вся система помещена в достаточно сильное магнитное поле, направленное вдоль оси $z$, замагничивающее движение электронов плазмы и пучка. В этом случае движение частиц становится одномерным. Динамика электронной подсистемы плазмы описывается системой гидродинамических уравнений:

$$
\begin{gathered}
\frac{\partial n_{p}}{\partial t}+\frac{\partial\left(n_{p} v_{p}\right)}{\partial z}=\frac{\partial n_{0}(t)}{\partial t}, \\
\frac{\partial v_{p}}{\partial t}+v_{p} \frac{\partial v_{p}}{\partial z}=-\frac{e}{m} E_{z} .
\end{gathered}
$$

Здесь $n_{p}$ и $v_{p}$ - концентрация и $z$-компонента скорости электронов плазмы, $e=|e|$ и $m-$ величина заряда и масса электрона, $E_{z}$ - компонента электрического поля. Правая часть уравнения непрерывности представляет собой источник изменения электронной концентрации. Линеаризуем систему (1) относительно малых возмущений исходного состояния, для чего представим концентрацию электронов плазмы в виде $n_{p}=n_{0}(t)+\delta n_{p}$, скорость $v_{p}=\delta v_{p}$ и электрическое поле $E_{z}$ в невозмущенном состоянии отсутствуют и, таким образом, имеют первый порядок малости. В результате получим

$$
\begin{gathered}
\frac{\partial \delta n_{p}}{\partial t}+n_{0}(t) \frac{\partial \delta v_{p}}{\partial z}=0 \\
\frac{\partial \delta v_{p}}{\partial t}=-\frac{e}{m} E_{z} .
\end{gathered}
$$

Динамика электронов пучка описывается системой, аналогичной (1):

$$
\begin{gathered}
\frac{\partial n_{b}}{\partial t}+\frac{\partial n_{b} v_{b}}{\partial z}=0 \\
\frac{\partial v_{b}}{\partial t}+v_{b} \frac{\partial v_{b}}{\partial z}=-\frac{e}{m \gamma^{3}\left(v_{b}\right)} E_{z} .
\end{gathered}
$$

Невозмущенная плотность пучка считается стационарной и учтены релятивистские эффекты в движении электронов пучка, $\gamma\left(v_{b}\right)=\left(1-v_{b}^{2} / c^{2}\right)^{-1 / 2}-$ релятивистский фактор, $c$ - скорость света в вакууме. Задавая возмущения плотности и скорости пучка в виде $n_{b}=n_{b 0}+\delta n_{b}, v_{b}=u+\delta v_{b}$, запишем линеаризованную относительно возмущений систему уравнений для электронного пучка

$$
\begin{gathered}
\frac{\partial \delta n_{b}}{\partial t}+u \frac{\partial \delta n_{b}}{\partial z}+n_{b 0} \frac{\partial \delta v_{b}}{\partial z}=0, \\
\frac{\partial \delta v_{b}}{\partial t}+u \frac{\partial \delta v_{b}}{\partial z}=-\frac{e}{m \gamma^{3}} E_{z},
\end{gathered}
$$

где $\gamma=\left(1-u^{2} / c^{2}\right)^{-1 / 2}$. Действие магнитного поля как сильного внешнего, так и поля волны сводится к „замораживанию“ движения электронов пучка и плазмы в поперечном направлении.

Динамика возбуждаемых электромагнитных полей описывается системой уравнений Максвелла. В рассматриваемой постановке полное электромагнитное поле может быть представлено в виде суперпозиции независимых между собой волн $E$ - и $B$-типа, имеющих отличную от нуля $E_{z}$ - и $B_{z}$-компоненту соответственно. Для целей возбуждения прямолинейным электронным пучком электромагнитных волн в условиях черенковского резонанса представляет интерес только волна $E$-типа, уравнение для $E_{z}$-компоненты которой имеет вид:

$$
\begin{aligned}
& \left(\frac{\partial^{2}}{\partial z^{2}}-k_{\perp}^{2}-\frac{1}{c^{2}} \frac{\partial^{2}}{\partial t^{2}}\right) \frac{\partial E_{z}}{\partial t} \\
& \quad=4 \pi\left(\frac{1}{c^{2}} \frac{\partial^{2}}{\partial t^{2}}-\frac{\partial^{2}}{\partial z^{2}}\right) j_{z}+A e^{-i \omega t} \delta(z) .
\end{aligned}
$$

Здесь $j_{z}$ - компонента электрического тока вдоль оси системы, в линейном приближении имеющая вид:

$$
j_{z}=-e n_{0}(t) \delta v_{p}-e\left(n_{b 0} \delta v_{b}+u \delta n_{b}\right) .
$$

Величина $k_{\perp}$ в (5) определяет поперечную структуру поля в системе. Для случая косого распространения волны эта зависимость имеет вид $\sim e^{i k_{x} x+i k_{y} y}$, где $k_{x}^{2}+k_{y}^{2}=k_{\perp}^{2}$. В прямоугольном волноводе поперечная структура поля определяется фактором $\sin \left(k_{x} x\right) \sin \left(k_{y} y\right)$, с $k_{x}=\pi n_{x} / L_{x}$ и $k_{y}=\pi n_{y} / L_{y}, n_{x, y}$ - целые числа, a $L_{x, y}$ - поперечные размеры волновода. В цилиндрическом волноводе радиальная и азимутальная зависимости имеют вид $\sim J_{l}\left(k_{\perp} r\right) e^{i l \varphi}$, где $k_{\perp}$ определяется нулями функции Бесселя $k_{\perp}=\mu_{l n} / R\left(J_{l}\left(\mu_{l n}\right)=0\right), l$ - целое число, $n$ - натуральное число. Последнее слагаемое в правой части (5) описывает сосредоточенный источник на частоте $\omega$ с амплитудой $A$ и соответствует заданию граничного условия для поля при $z=0$. Предполагаем, что этот источник включен хотя и в далеком, но конечном прошлом, и, таким образом, при $z \rightarrow \pm \infty$ возмущения убывают даже для усиливающейся волны. Формально это можно реализовать, если считать, что частота $\omega$ имеет достаточно большую положительную мнимую часть. Случай вещественных значений $\omega$ может быть получен аналитическим продолжением из верхней полуплоскости комплексной плоскости $\omega$ на вещественную ось. Кроме того, предполагается, что пучок входит через плоскость $z=0$ невозмущенным как по скорости, так и по плотности.

Рассмотрим задачу усиления сигнала, подаваемого на вход системы при $z=0$. Будем предполагать, что решения в рассматриваемой области $z>0$ определяются только условиями при $z=0$. Это реализуется на достаточно больших временах $t$, когда все возмущения в начальных условиях уже „вынесены“ за пределы зоны усиления. Применим к системе (2), (4)-(6) преобразование Фурье по координате $z$

$$
f\left(t, k_{z}\right)=\int_{-\infty}^{+\infty} f(t, z) e^{-i k_{z} z} d z
$$

и, сохраняя за образами аналогичные обозначения, получим систему уравнений, описывающую нестационарные 
процессы в усилителе:

$$
\begin{gathered}
\frac{\partial \delta n_{p}}{\partial t}+i k_{z} n_{0}(t) \delta v_{p}=0 \\
\frac{\partial \delta n_{b}}{\partial t}+i k_{z}\left(u \delta n_{b}+n_{b 0} \delta v_{b}\right)=0 \\
\frac{\partial \delta v_{p}}{\partial t}=F, \quad \frac{\partial \delta v_{b}}{\partial t}+i k_{z} u \delta v_{b}=F \gamma^{-3}, \\
\left(k_{z}^{2}+k_{\perp}^{2}+\frac{1}{c^{2}} \frac{\partial^{2}}{\partial t^{2}}\right) \frac{\partial F}{\partial t} \\
=\frac{4 \pi e}{m}\left(\frac{1}{c^{2}} \frac{\partial^{2}}{\partial t^{2}}+k_{z}^{2}\right) j_{z}+A e^{-i \omega t} .
\end{gathered}
$$

В (8) введено обозначение $F=-e E_{z} / m$, плотность тока определяется соотношением (6).

Система (8) не может быть решена аналитически за исключением случая $n_{0}(t)=$ const. Если плотность плазмы меняется медленно, то решение системы (8) следует искать методом теории возмущений в виде

$$
f\left(t, k_{z}\right)=\left(f_{0}\left(t, k_{z}\right)+f_{1}\left(t, k_{z}\right)\right) e^{-i \omega t},
$$

где через $f$ обозначена совокупность неизвестных функций системы (8). Входящие в (9) амплитуды $f_{0}\left(t, k_{z}\right)$ и $f_{1}\left(t, k_{z}\right)$ имеют различный порядок малости по параметру $\varepsilon=T / \tau \ll 1$, где $T-$ период колебаний, а $\tau$ - характерное время изменения плотности плазмы. А именно $f_{0}\left(t, k_{z}\right)$ имеет нулевой порядок малости $\sim \varepsilon^{0}$, а $f_{1}\left(t, k_{z}\right)-$ первый $\sim \varepsilon^{1}$, при этом обе амплитуды являются медленными функциями времени, т.е. $f_{0,1}\left(t, k_{z}\right)=f_{0,1}\left(\varepsilon t, k_{z}\right)$. Более высокими (выше первого) порядками малости будем пренебрегать.

В нулевом порядке малости система (8) сведется к уравнению для амплитуды $F_{0}\left(t, k_{z}\right)$

$$
\begin{aligned}
& \left\{k_{\perp}^{2}+\left(k_{z}^{2}-\frac{\omega^{2}}{c^{2}}\right)\right. \\
& \left.\quad \times\left(1-\frac{\omega_{p}^{2}(t)}{\omega^{2}}-\frac{\omega_{b}^{2} \gamma^{-3}}{\left(\omega-k_{z} u\right)^{2}}\right)\right\} F_{0}\left(t, k_{z}\right)=i A / \omega .
\end{aligned}
$$

В (10) введены ленгмюровские частоты электронов плазмы и пучка $\omega_{p}^{2}(t)=4 \pi n_{0}(t) e^{2} / m$ и $\omega_{b}^{2}=4 \pi n_{b 0} e^{2} / m$. Выражение в фигурных скобках (10) (обозначим как $\left.D_{p b}\right)$ есть дисперсионная функция, равенство нулю которой представляет собой хорошо известное дисперсионное уравнение плазма-пучковой системы [19]. При заданной частоте сигнала оно определяет четыре распространяющиеся волны, одна из которых имеет отрицательную мнимую часть $\operatorname{Im} k_{z}$ и соответствует усиливаемой в положительном направлении оси $z$ волне. Максимум пространственного инкремента неустойчивости $\left|\operatorname{Im} k_{z}\right|$ при $\omega_{b} \ll \omega_{p}$ реализуется при частоте, близкой к резонансной, определяемой из условий

$$
k_{\perp}^{2}+\left(k_{z}^{2}-\frac{\omega^{2}}{c^{2}}\right)\left(1-\frac{\omega_{p}^{2}(t)}{\omega^{2}}\right)=0, \omega-k_{z} u=0 .
$$

При уменьшении частоты сигнала от резонансной инкремент монотонно убывает до нуля при $\omega \rightarrow 0$. Наоборот, с ростом частоты сигнала инкремент быстро падает до нуля и неустойчивость исчезает. Из приведенных рассуждений может быть определено и влияние временной динамики плотности плазмы на пучково-плазменную неустойчивость. С ростом концентрации электронов по отношению к оптимальной (дающей максимальное значение $\left.\left|\operatorname{Im} k_{z}\right|\right)$, инкремент плавно снижается, при уменьшении концентрации электронов плазмы инкремент резко падает до нуля.

Далее в первом порядке малости из системы (8) может быть получено выражение

$$
\begin{aligned}
& F_{1}\left(t, k_{z}\right)=-\frac{i}{\omega D_{p b}}\left(k_{\perp}^{2}+k_{z}^{2}-3 \frac{\omega^{2}}{c^{2}}+\left(k_{z}^{2}+\frac{\omega^{2}}{c^{2}}\right) \frac{\omega_{p}^{2}(t)}{\omega^{2}}\right. \\
& \left.+\left(k_{z}^{2}\left(\omega+k_{z} u\right)+\frac{\omega^{2}}{c^{2}}\left(\omega-3 k_{z} u\right)\right) \frac{\omega_{b}^{2} \gamma^{-3}}{\left(\omega-k_{z} u\right)^{2}}\right) F_{0}^{\prime}\left(t, k_{z}\right) \\
& +2 F_{0}\left(t, k_{z}\right) \frac{\partial \omega_{p}^{2}(t)}{\partial t} .
\end{aligned}
$$

Малость $F_{1}\left(t, k_{z}\right)$ по сравнению с $F_{0}\left(t, k_{z}\right)$ обеспечивается малостью производной $\partial \omega_{p}^{2}(t) / \partial t$, которой и пропорциональна зависимость $F_{1}\left(t, k_{z}\right)$. Для восстановления решения $F(t, z)$ выполним обратное преобразование Фурье

$$
F(t, z)=\frac{1}{2 \pi} \int_{-\infty}^{+\infty}\left(F_{0}\left(t, k_{z}\right)+F_{1}\left(t, k_{z}\right)\right) e^{i k_{z} z} d k_{z} .
$$

Для вычисления $F(t, z)$ при $z>0$ замкнем контур интегрирования полуокружностью бесконечно большого радиуса в области $\operatorname{Im} k_{z}>0$. Интеграл по этой полуокружности равен нулю. Тогда вычисление интеграла (13) сведется к вычислению вычетов подынтегральной функции в особых точках - корнях дисперсионного уравнения. Согласно выражению $(10), F_{0}\left(t, k_{z}\right)$ имеет четыре полюса первого порядка. Выражение $F_{1}\left(t, k_{z}\right)$ в тех же точках комплексной плоскости $k_{z}$ имеет полюса третьего порядка. Обозначим их $k_{n}(t)$, где $n=1 \ldots 4$. Направления распространения волн, соответствующих $k_{n}(t)$, определяются по положению этих величин на комплексной плоскости $k_{z}$ при достаточно больших значениях $\operatorname{Im} \omega>0$ [20]. При $\operatorname{Im} \omega \rightarrow+\infty$ имеем предельные выражения

$$
k_{1,2} \rightarrow i \frac{\operatorname{Im} \omega}{u}, \quad k_{3,4} \rightarrow \pm i \frac{\operatorname{Im} \omega}{c} .
$$

Волна, соответствующая $k_{4}$, распространяется в сторону, противоположную направлению движения частиц пучка, три других волны - попутные с пучком. Таким образом, внутри построенного контура интегрирования лежат три полюса $k_{1,2,3}(t)$. При вещественной $\omega$ в области частот, где развивается неустойчивость, одно 
из $k_{1,2,3}(t)$ - вещественное и два комплексно-сопряженных, т.е. попутными с пучком будут одна волна, распространяющаяся без усиления и затухания, одна затухающая и одна - усиливающаяся. В итоге $F_{0}(t, z)$ может быть представлено в виде

$$
F_{0}(t, z)=C_{1}(t) e^{i k_{1}(t) z}+C_{2}(t) e^{i k_{2}(t) z}+C_{3}(t) e^{i k_{3}(t) z} .
$$

Вычисление коэффициентов $C_{n}(t)$ не представляет принципиальных трудностей, однако их запись в явном аналитическом виде чрезвычайно громоздка и не приводится. Каждое из слагаемых (15) представляет собой нормальную моду колебаний. Заданные на границе поля приводят к возбуждению всей совокупности нормальных мод, однако в процессе распространения и усиления нормальных мод доминирующей окажется усиливающаяся мода. Пусть усилению волны соответствует волновое число $k_{1}$, а усиливающейся волне - первое слагаемое в (15). На достаточно больших расстояниях $z$ (при рассматриваемых в настоящей работе параметрах речь идет о нескольких длинах волн) функция

$$
\begin{aligned}
F_{0}(t, z) & \approx C_{1}(t) e^{i k_{1}(t) z} \\
& =\left|C_{1}(t)\right| e^{\left|k_{1}^{\prime \prime}(t)\right| z} e^{i k_{1}^{\prime}(t) z+i \operatorname{arctg}\left(C_{1}^{\prime \prime}(t) / C_{1}^{\prime}(t)\right)},
\end{aligned}
$$

где ' и " означают вещественную и мнимую части комплексного числа. Переменная во времени фаза

$$
\varphi(t)=\varphi_{1}(t)+\varphi_{2}(t)=k_{1}^{\prime}(t) z+\operatorname{arctg}\left(C_{1}^{\prime \prime}(t) / C_{1}^{\prime}(t)\right)
$$

определяет смещение частоты

$$
\Delta \omega=\Delta \omega_{1}+\Delta \omega_{2}=-d \varphi_{1}(t) / d t-d \varphi_{2}(t) / d t .
$$

Два слагаемых в (17) описывают два различных механизма изменения частоты усиливаемого сигнала. Первый соответствует ситуации, когда в точке $z$ формируется набег фазы, изменяющийся во времени [21]. Второй механизм связан с тем, что граничные условия приводят к возбуждению всей совокупности нормальных мод, причем комплексная амплитуда той или иной моды определяется параметрами системы. В случае меняющихся параметров это также приводит к появлению переменной во времени фазы. Набег фазы за счет первого механизма пропорционален расстоянию, проходимому усиливающейся волной. Если $k_{1}^{\prime} \neq 0$, то при достаточно большой длине системы он окажется доминирующим. В реальных системах плазменной СВЧ электроники длина пространства взаимодействия электронного пучка с плазмой составляет порядка 10 длин волн. При таких относительно небольших длинах оба механизма смещения частоты могут оказаться конкурирующими между собой.

По поводу знака $\Delta \omega$ отметим следующее обстоятельство. Поскольку вся зависимость от времени в (18) входит через зависимость $n_{0}(t)$, формула (18) может быть переписана в виде

$$
\Delta \omega=-\frac{d \varphi\left(n_{0}(t)\right)}{d n_{0}} \frac{d n_{0}(t)}{d t} .
$$

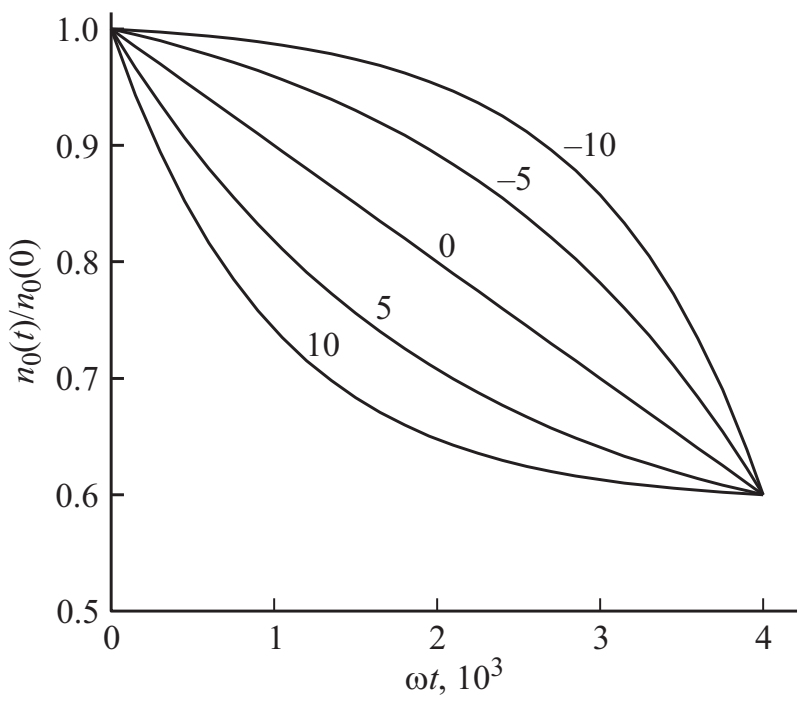

Рис. 1. Временная зависимость уменьшающейся плотности плазмы для различных значений параметра $\beta=0, \pm 5, \pm 10$.

Различные направления изменения плотности плазмы со временем (увеличение/уменьшение концентрации электронов) будут приводить к противоположным между собой знакам $\Delta \omega$.

Для численного анализа соотношения (18) возьмем параметры, близкие к используемым в экспериментах по плазма-пучковому взаимодействию $[1,2]$. Зададим временную зависимость плотности электронов плазмы в виде

$$
n_{0}(t)=n_{0}(0)\left(1 \mp \frac{t_{f}}{\tau} \frac{1-\exp (-\beta t / \tau)}{1-\exp \left(-\beta t_{f} / \tau\right)}\right),
$$

где $t_{f}$ - время, в течение которого производится наблюдение, и к этому моменту времени плотность плазмы изменяется в $1 \mp t_{f} / \tau$ раз. Знак „-“ соответствует уменьшающейся плотности плазмы, а знак „+“ - увеличивающейся. Параметр $\beta$ характеризует форму временного профиля (20), в частности, при $\beta=0$ имеем линейный закон изменения плотности плазмы со временем. Выберем ленгмюровские частоты плазмы и пучка $\omega_{p}(0) / \omega=1.5$ и $\omega_{b} \gamma^{-3 / 2} / \omega=0.3$, характерное время изменения плотности плазмы $\omega \tau=10^{4}$, длину системы $z / u T=7$ и время $\omega t_{f}=4 \cdot 10^{3}$, характерное значение поперечного волнового числа $k_{\perp} u / \omega=1$. Взятые параметры соответствуют ситуации, когда плотность плазмы заметно меняется на временах в несколько сотен периодов колебаний, а на длине системы укладывается 7 длин волн. К моменту времени $t_{f}$ плотность плазмы изменяется на $40 \%$.

На рис. 1 представлены зависимости от времени плотности плазмы для различных $\beta$, значение которых указано около каждой кривой. Во всех случаях плотность плазмы убывает. На рис. 2 для случая $\beta=0$ представлена временная динамика инкремента неустойчивости $\left|k_{1}^{\prime \prime}(t)\right|$ (сплошная кривая) и величины 


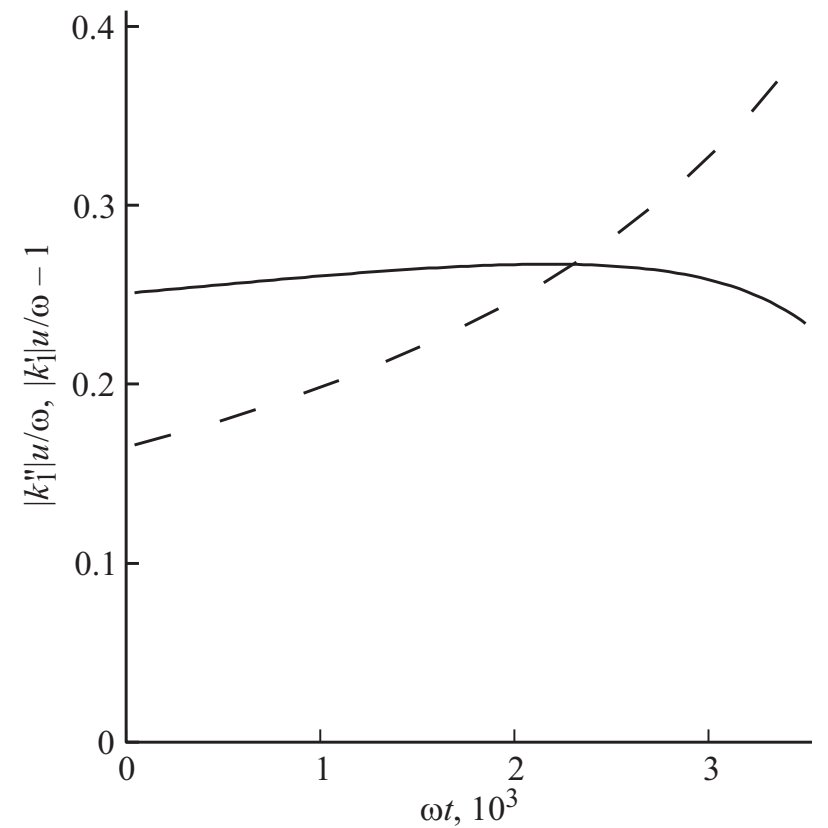

Рис. 2. Временная зависимость пространственного инкремента неустойчивости (сплошная кривая) и вещественной части волнового числа усиливаемой волны (штриховая кривая) для $\beta=0$.

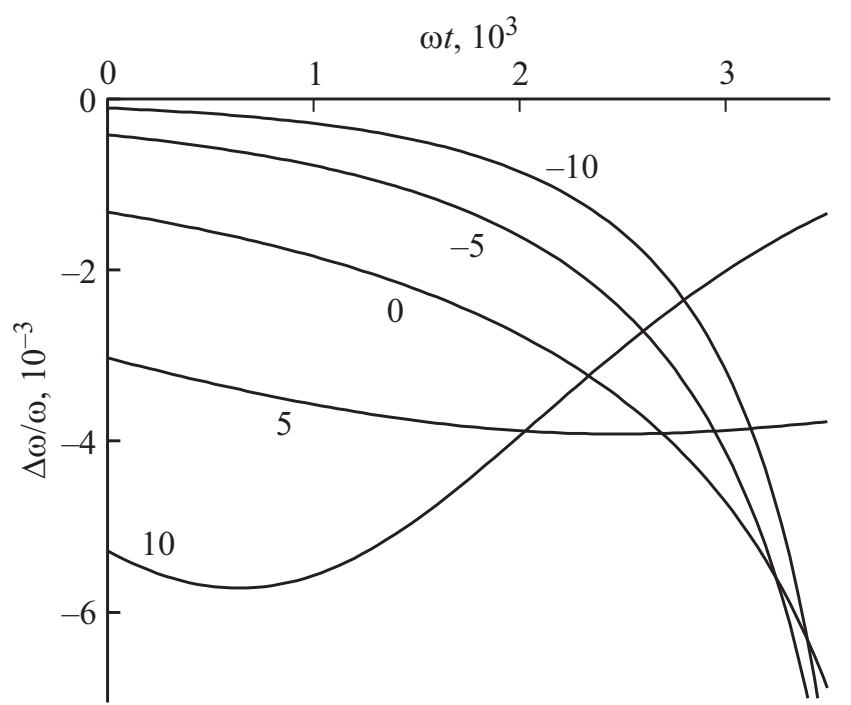

Рис. 3. Временная зависимость смещения частоты усиливаемого сигнала при уменьшающейся плотности плазмы для различных значений параметра $\beta=0, \pm 5, \pm 10$.

$k_{1}^{\prime}(t)-\omega / u$ (штриховая кривая), характеризующей переменный во времени набег фазы. Параметры выбраны таким образом, что вначале плотность плазмы больше оптимального значения, при котором реализуется максимум пространственного усиления, а затем в течение импульса она снижается до значения меньше оптимального. Вместе с $\left|k_{1}^{\prime \prime}(t)\right|$ изменяется и $k_{1}^{\prime}(t)$, что формирует переменный во времени набег фазы волны на выходе из усилителя. Более значительным оказывается смещение частоты, порождаемое переменным во времени набегом фазы на выходе из усилителя. Аналогичная картина имеет место и при других значениях $\beta$, а также в случае увеличения плотности плазмы. Далее на рис. 3 представлены временные зависимости суммарного (за счет обоих эффектов) изменения частоты усиливаемого сигнала $\Delta \omega(t) / \omega$ для временных профилей плотности плазмы, как на рис. 1. При уменьшении $\beta$ от 10 до -10 характер изменения плотности плазмы меняется: быстрое изменение в начале импульса и медленное в конце сменяется на медленное изменение в начале и быстрое

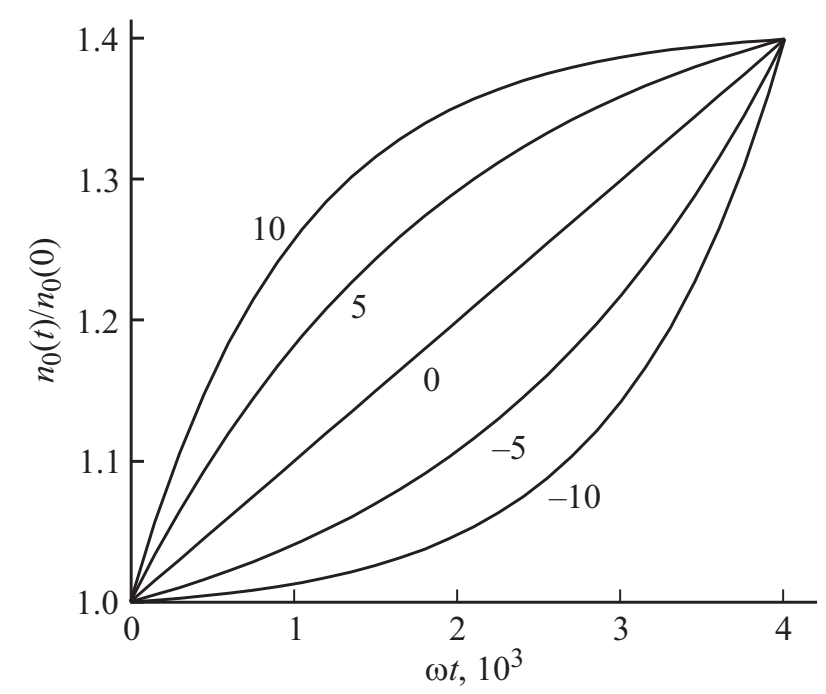

Рис. 4. Временная зависимость увеличивающейся плотности плазмы для различных значений параметра $\beta=0, \pm 5, \pm 10$.

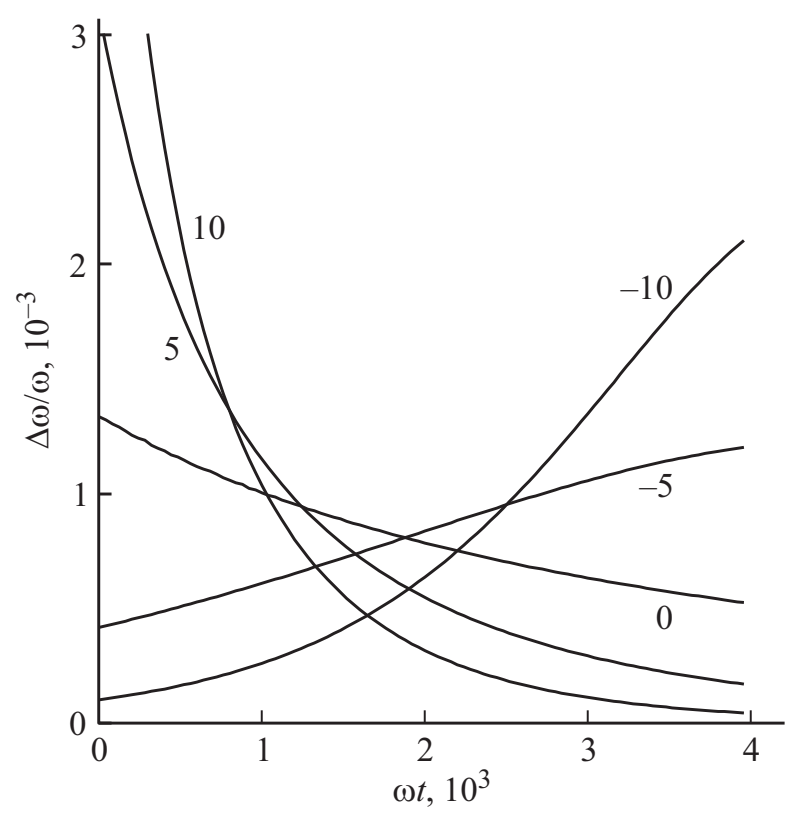

Рис. 5. Временная зависимость смещения частоты усиливаемого сигнала при увеличивающейся плотности плазмы для различных значений параметра $\beta=0, \pm 5, \pm 10$. 
в конце импульса. В силу (19), это определяет и характер временной динамики смещения частоты сигнала. В начале импульса при положительных значениях $\beta$ плотность электронов плазмы меняется быстро и смещение частоты значительное. При переходе в область отрицательных значений $\beta$ имеем медленное изменение концентрации электронов плазмы в начале импульса и уменьшение величины $|\Delta \omega|$. В конце импульса характер изменения плотности плазмы меняется на обратный и зависимость $\Delta \omega$ от $\beta$ также меняется на обратную.

На рис. 4 и 5 показаны аналогичные рис. 1 и 3 зависимости для растущей плотности плазмы (знак „, + “ в формуле (20)). Здесь также следует отметить разный характер зависимости $\Delta \omega$ от $\beta$ вначале и в конце импульса, что в полной мере объясняется зависимостью $\Delta \omega$ от скорости изменения концентрации электронов плазмы (19). Что касается знака $\Delta \omega$ на рис. 3 и 5, то он имеет противоположный характер для уменьшающейся и увеличивающейся плотности плазмы в соответствии со знаком $d n_{0} / d t$ в (19).

Таким образом, изменение частоты усиливаемого при пучково-плазменной неустойчивости сигнала, обусловленное весьма незначительной нестационарностью плазмы, оказывается существенным и вполне может быть измерено при помощи современных экспериментальных методов [21]. Это обстоятельство следует принимать во внимание при исследовании плазменных СВЧ излучателей, особенно их спектральных характеристик.

\section{Список литературы}

[1] Стрелков П.С., Иванов И.Е., Шумейко Д.В. // Физика плазмы. 2012. Т. 38. № 6. С. 536-543. [Strelkov P.S., Ivanov I.E., Shumeiko D.V. // Plasma Phys. Rep. 2012. Vol. 38. N 6. P. 488-495.]

[2] Стрелков П.С., Тараканов В.П., Иванов И.Е., Шумейко Д.В. // Физика плазмы. 2014. Т. 40. № 8. С. 738-748. [Strelkov P.S., Tarakanov V.P., Ivanov I.E., Shumeiko D.V. // Plasma Phys. Rep. 2014. Vol. 40. N 8. P. 640-649.]

[3] Ульянов Д.К., Баранов Р.В., Лоза О.Т., Ернылева С.Е., Богданкевич И.Л. // ЖТФ. 2013. Т. 83. Вып. 10. С. 113116. [Ul'yanov D.K., Baranov R.V., Loza O.T. et al. // Tech. Phys. 2013. Vol. 58. N 10. P. 1503-1506.]

[4] Лоза О.Т., Ульянов Д.К., Баранов Р.В. // ЖТФ. 2011. Т. 81. Вып. 3. C. 98-102. [Loza O.T., Ulyanov D.K., Baranov R.V. // Tech. Phys. 2011. Vol. 56. N 3. P. 413-417.]

[5] Стрелков П.С., Тараканов В.П., Иванов И.Е., Шумейко Д.В. // Физика плазмы. 2015. Т. 41. № 6. С. 533-541. [Strelkov P.S., Tarakanov V.P., Ivanov I.E., Shumeiko D.V. // Plasma Phys. Rep. 2015. Vol. 41. N 6. P. 492-500.]

[6] Карташов И.Н., Кузелев М.В., Рухадзе А.А., Сепехри Джсаван Н. // ЖТФ. 2005. Т. 75. Вып. 3. C. 15-23. [Kartashov I.N., Kuzelev M.V., Rukhadze A.A., Sepehri Javan N. // Tech. Phys. 2005. Vol. 50. N 3. P. 298307.]

[7] Карташов И.Н., Кузелев М.В., Сепехри Джсаван Н. // Физика плазмы. 2005. T. 31. № 3. C. 274-282. [Kartashov I.N., Kuzelev M.V., Sepehri Javan N. // Plasma Phys. Rep. 2005. Vol. 31. N 3. P. 244-252.]
[8] Картамов И.Н., Кузелев М.В., Рухадзе А.А. // ЖТФ. 2006. T. 76. № 2. C. 10-20. [Kartashov I.N., Kuzelev M.V., Rukhadze A.A. // Tech. Phys. 2006. Vol. 51. N 2. P. 151-162.]

[9] Карташов И.Н., Кузелев М.В., Рухадзе А.А. // Физика плазмы. 2009. Т. 35. № 2. С. 194-201. [Kartashov I.N., Kuzelev M.V., Rukhadze A.A. // Plasma Phys. Rep. 2009. Vol. 35. N 2. P. 169-176.]

[10] Тараканов В.П., Шустин Е.Г. // Физика плазмы. 2007. T. 33. № 2. C. 151-158. [Tarakanov V.P., Shustin E.G. // Plasma Phys. Rep. 2007. Vol. 33. N 2. P. 130-137.]

[11] Гинзбург В.Л., Франк И.М. // ЖЭТФ. 1946. Т. 16. № 1. C. $15-29$.

[12] Гинзбург В.Л. // Известия вузов. Радиофизика. 1973. Т. 16. № 4. C. 512-516. [Ginzburg V.L. // Radiophys. Quant. Electron. 1973. Vol. 16. N 4. P. 386-389.]

[13] Гинзбург В.Л., Цытович В.Н. // ЖЭТФ. 1974. Т. 65. № 1. C. 132-144. [Ginzburg V.L., Tsytovich V.N. // Sov. Phys. JETP. 1974. Vol. 38. N 1. P. 65-70.]

[14] Ерохин Н.С., Моисеев С.С., Назаренко Л.А. // ЖЭТФ. 1975. T. 69. № 1. C. 131-141. [Erokhin N.S., Moiseev S.S., Nazarenko L.A. // Sov. Phys. JETP. 1975. Vol. 42. N 1. P. 67 72.]

[15] Шварибург А.Б. // УФН. 2000. Т. 170. № 12. С. $1297-$ 1324. [Shvartsburg A.B. // Phys.-Uspekhi. 2000. Vol. 43. N 12. P. 1201-1228.]

[16] Шварибург А.Б. // УФН. 2005. Т. 175. № 8. С. 833861. [Shvartsburg A.B. // Phys.-Uspekhi. 2005. Vol. 48. N 8. P. 797-823.]

[17] Шварибург А.Б. // Квантовая электроника. 1998. Т. 25. № 3. C. 201-205. [Shvartsburg A.B. // Quant. Electron. 1998. Vol. 28. N 3. P. 193.]

[18] Rostomyan E.V. // Plasma Phys. Control. Fusion. 2008. Vol. 50. P. 085019.

[19] Кузелев М.В., Рухадзе А.А., Стрелков П.С. Плазменная релятивистская СВЧ электроника. М.: Изд-во МГТУ им. Н.Э. Баумана, 2002.

[20] Кузелев М.В., Рухадзе А.А. Методы теории волн в средах с дисперсией. М.: Физматлит, 2007. [Kuzelev M.V., Rukhadze A.A. Methods of wave theory in dispersive media. World Scientific, 2010.]

[21] Карташов И.Н., Кузелев М.В., Стрелков П.С., Тараканов В.П. // Физика плазмы. 2018. Т. 44. № 2. С. 250-260. [Kartashov I.N., Kuzelev M.V., Strelkov P.S., Tarakanov V.P. // Plasma Phys. Rep. 2018. Vol. 44. N 2. P. 289-298.] 